\title{
Mechanobiological conceptual framework for assessing stem cell bioprocess effectiveness
}

\author{
Mee-Hae $\mathrm{Kim}^{1}$ and Masahiro Kino-oka ${ }^{1}$ \\ ${ }^{1}$ Osaka University
}

May 25, 2021

\begin{abstract}
The realization of the enormous potential of stem cells requires development of efficient bioprocesses and optimization drawing drawn from mechanobiological considerations. Here, we emphasize the importance of mechanotransduction as one of the governing principles of stem cell bioprocesses, underscoring the need to further explore the behavioral mechanisms involved in sensing mechanical cues and coordinating transcriptional responses. We identify the sources of the intrinsic, extrinsic, and external noise in bioprocess under uncertainty, and discuss criteria and indicators that might assess and predict cellto-cell variability resulting from environmental fluctuations. Specifically, we propose a conceptual framework to explain the impact of mechanical forces within cellular environment and identify key cell state determinants in bioprocess and discuss their implementation challenges.
\end{abstract}

Mechanobiological conceptual framework for assessing stem cell bioprocess effectiveness

Mee-Hae Kim* and Masahiro Kino-oka

Department of Biotechnology, Graduate School of Engineering, Osaka University, 2-1 Yamadaoka, Suita, Osaka 565-0871, Japan

* Correspondence

Mee-Hae Kim, Department of Biotechnology, Graduate School of Engineering, Osaka University, 2-1 Yamadaoka, Suita, Osaka 565-0871, Japan

Email: mh-kim@bio.eng.osaka-u.ac.jp

Tel.: +81 (0)6 6879 7445; Fax: +81 (0)6 68794246

Running title: Mechanobiological Conceptual Framework Development

\section{Abstract}

The realization of the enormous potential of stem cells requires development of efficient bioprocesses and optimization drawing drawn from mechanobiological considerations. Here, we emphasize the importance of mechanotransduction as one of the governing principles of stem cell bioprocesses, underscoring the need to further explore the behavioral mechanisms involved in sensing mechanical cues and coordinating transcriptional responses. We identify the sources of the intrinsic, extrinsic, and external noise in bioprocess under uncertainty, and discuss criteria and indicators that might assess and predict cell-to-cell variability resulting from environmental fluctuations. Specifically, we propose a conceptual framework to explain the impact of mechanical forces within cellular environment and identify key cell state determinants in bioprocess and discuss their implementation challenges. 
KEYWORDS: stem cell bioprocessing, conceptual framework, mechanotransduction, intrinsic and extrinsic noise, intrinsic disorder of bioprocess; cell mechanobiology

\section{Complexity of Stem Cell Bioprocess Design: The Need to Consider Cellular Mechanics}

Stem cells have a remarkable ability to undergo self-renewal in an undifferentiated state and differentiation into one or more cell types (Pittenger et al., 1999a; Tewary et al., 2018). These cell types include embryonic stem cells (ESCs) that are derived from the inner cell mass of blastocysts; mesenchymal stem cells (MSCs) that originate from adult tissues; and induced pluripotent stem cells (iPSCs) that can be reprogrammed into their pluripotent state by treatment with defined factors from a somatic cell (Thomson el al., 1998; Yamanaka, 2020; Zhu et al., 2021). Recent stem cell-based technologies have substantially accelerated the applicability of these cells in disease modeling and cell-replacement therapy (Harrison et al., 2018; Madl et al., 2018; McKee and Chaudhry, 2017; Pittenger et al., 1999b). The capacity to develop potential stem cell applications will rest on the ability of users to process large numbers of cells. In vitro stem cell maintenance and propagation constitutes a major technical challenge due to fluctuations in cell properties and complex relationship between culture conditions and process outcomes (Kami et al., 2013; Kim and Kino-oka, 2020b; Mount et al., 2015; Panchalingam et al., 2015; Thomas et al., 2008). Especially, the importance of mechanical force has been extensively studied in single cells, but the behavior of multiple cells is less well understood, largely because of the limited generation of mechanical forces in maintenance of cellular homeostasis. Stem cells monitor their physical and mechanical environment via macromolecular complexes, known as mechanosensors, and initiate an adaptive response in an unfavorable mechanical environment (Figure 1 ). It has recently been suggested that the cell nucleus is exposed to the mechanical forces transmitted through the actin cytoskeleton from outside the cell, and the changes in nuclear morphology possibly affect the regulation of cellular homeostasis to maintain self-renewal and pluripotency (Discher et al., 2009; Dupont et al., 2011; Ingber, 1997; Iskratsch et al., 2014). In particular, cells remember their behavioral changes in past environments. For instance, it has been shown that mechanotransduction through cytoskeletal contractility, Rho family GTPase signaling, and subsequent changes in epigenetic marks, such as DNA methylation, histone modifications, and chromatin state, play major roles in cellular homeostasis (Matthews et al, 2006; Rosowski et al., 2015; Thanuthanakhun et al., 2021). The ability of cells to maintain a constant level of cytoskeletal tension in response to external and internal disturbances is defined by tensional homeostasis (Ambriz et al., 2018; Lenormand et al., 2007; Mizuno et al., 2007; Walker et al., 2020a; Webster et al., 2014). In response to the fluctuating nature of a bioprocess, cells can generate internal forces either by extending membranes or by rearranging their actin cytoskeletons, thereby producing contractile forces. In these processes, the breakdown in tensional homeostasis can be caused by alterations in the biological processes involved in force sensing and conversion, cell adhesion molecules such as integrin and cadherin, cell cytoskeleton, and various cytoplasmic and nuclear signaling molecules. The breakdown in tensional homeostasis can lead to changes in epigenetic state/memory, thereby leading to functional variability in stem cells (Alisafaei et al., 2019).

The field of stem cell mechanobiology is rapidly developing with the growing realization of the importance of biophysical and biomechanical factors and is being adopted in the development of new bioengineering technologies (Argentati et al., 2019; Ingber, 2018; Mammoto et al., 2013; Sun et al., 2012; Roca-Cusachs et al., 2017). Many studies have focused on the development of culture strategies that can recapitulate the physico-chemical properties of the complex native cellular microenvironment (Jo et al., 2020; Li et al., 2021). A large effort has been made over the last decades to propose various strategies to develop affordable high-quality cell-based products in a reproduceable and robust manner (Kim and Kino-oka, 2018; Kim and Kino-oka, 2020a). However, a knowledge gap that still exists in the mechanobiological sciences for the stem cell bioprocess development, optimization and characterization is not currently being filled by the bioprocess research. In addition, no straightforward means exist for the development of new techniques, such as bioprocess equipment operations; process design, optimization, and scale-up; and monitoring, analysis, and prediction. The creation of a conceptual framework as a guide for the strategies involved in bioprocess development and optimization would be a necessary step towards full-fledged bioprocess integration that would serve to eliminate the adverse pressures currently faced by many bioengineers. 


\section{Mechanobiological Understanding of Internal and External Variability-Generated Uncertainty}

in Stem Cell Bioprocesses

Understanding the complex interplay between internal and external forces affecting cellular mechanotransduction under correctly combined conditions could be of benefit for optimal bioprocess design.Figure 2 illustrates the intrinsic disorder characteristics of stem cell bioprocesses dominated by external forcing and internal variability. Cellular functions are influenced not only by internal cellular machinery but also by external mechanical cues from the surrounding microenvironment. Forces that arise in biological systems can be classified into two main categories: internal and external (Chen et al., 2019; Mao and Baum, 2015; Vining and Mooney, 2017). Internal forces are defined as forces generated by cells themselves. The ability of cells to adhere, move, and divide requires a cytoskeleton that can reversibly assemble into a wide range of structures. The spatiotemporally organized forces produced between cells and their extracellular environment, as well as intercellular forces within cell colonies, play pivotal roles in driving these migration steps (Shuzui et al., 2019). In this way, the regulated re-modeling of cell-substrate and cell-cell interactions during growth aids the maintenance of cellular homeostasis. Conversely, external forces are defined as forces, such as tensile, compressive, or shear stresses, which are applied to cells from their environment and culture operation (Goodwin and Nelson, 2021; Ingber, 2018; Wall et al., 2018). These mechanical forces resulting from both intracellularly-generated and externally-applied forces can alter the internal equilibrium state, thereby affecting cellular mechanobiological responses. Experimental and theoretical investigations have determined the architecture of external noise and its causal factors, though substantial uncertainty surrounds the importance of mechanical forces (Charras and Yap, 2018; Ivanovska et al., 2015; Jégou and Romet-Lemonne, 2021). When subjected to mechanical forces, cells adopt a mechanoprotective and adaptative behavior, mechanically explained by a strain-stiffening process, to control membrane integrity, cell shape, and structural integrity (Doss et al., 2020; Stricker et al., 2010; Walker et al., 2020b). The situation is fundamentally different in signaling and regulatory networks. Interactions embedded in such signaling networks need to be extremely dynamic and versatile to be able to respond quickly to specific stimuli and to adapt to this response over time (Kinney et al., 2019; Mack et al., 2004; Mendez and Janmey, 2012). If the response time is too short, these forces are not transmitted sufficiently, and if it is too long, these forces are transmitted across the cytoskeleton to the nucleus, where they result in substantial deformation. These forces and deformations can regulate chromatin structure and transcriptional activity through a number of mechanisms (Miroshnikova et al., 2017; Tajik et al., 2016). Both magnitude and frequency of an external force affect the consequence (Freund et al., 2012; Kaazempur Mofrad et al., 2005; Mack et al., 2004). Many questions concerning the generation of internal and external mechanical forces in gene expression and their effects on cellular behavior remain unanswered. Since all cellular processes, including transcription and translation, are basic to stochasticity in biochemical processes, the fluctuations in the amount and state of mRNA and corresponding protein levels will influence their cellular functions (Raser and O'shea, 2005; Thomase et al., 2018). The mechanisms by which externally applied mechanical forces control stem cells during culture do not involve the direct and spontaneous triggering of differentiation per se, but rather the formation of a particular behavioral memory under dynamic microenvironment that can generate several mechanical stimuli. Importantly, there is the added complexity of demonstrating that the key process input variables are critical for the resultant product quality in a combinatorial process. In process in relation to a combinatorial operation, it is very difficult to determine cause-and-effect interactions and their relationship to the final product quality, since inputs may act singly, agonistically, or cumulatively on process outputs and final process output is a viable cell that cannot currently be comprehensively defined.

\section{Identifying Criteria and Indicators for Assessing External Forcing and Internal Variability- Generated Uncertainly in Stem Cell Bioprocesses}

Understanding the prevalence of pre-existing intrinsic and extrinsic variability over external noise is important for developing key indicators to identify and predict the bioprocess variability. The phenomenon of "intrinsic disorder" has been widely studied in bioprocess conditions, and understanding and controlling the sources of variability are anticipated to play important roles in improving bioprocess control and optimization (Kim and Kino-oka, 2020b; Misener et al., 2018). Indeed, it has been shown that the phenotypic variability among stem 
cells can contribute to the inherent differences resulting from donor-dependent variability as well as changes induced during cellular reprogramming and expansion and cell handling processes such as passaging, freezing, or thawing (Kim and Kino-oka, 2018). The causes of cell variability occurring in a series of bioprocessing steps and the related mechanisms are often still a "black box."

While the impact of forces and mechanical environment on the structure and function of stem cells has long been appreciated (Ivanovska et al., 2015; Liu et al., 2010; Maul et al., 2011; Mendelson and Frenette, 2014; Naqvi and McNamara, 2020), the importance of mechanical forces in bioprocessing has only recently been investigated. The potential sources of cell-to-cell variability to bioprocesses - described here as intrinsic (intra-cellular) noise, extrinsic (inter-cellular) noise, or external (environmental) noise - have been difficult to investigate because the evidence assessing the effect of external environmental fluctuations on a system is limited (Hilfinger and Paulsson, 2011). The intrinsic noise often refers to the inherent stochasticity of biochemical processes, such as transcription and translation (Raser and O'shea, 2005; Lei, 2009; Soltani et al., 2016; Swain et al. 2002; Thomas, 2019). In contrast, the extrinsic noise and external noise are generated form cellular processes, such as cell-cell and cell-matrix contacts, and cell migration or form environmental fluctuation, respectively (Lei, 2009; Hilfinger and Paulsson, 2011; Swain et al. 2002). Thus, it is essential to identify all factors causing intrinsic disorder within a bioprocess, based on the noise sources associated with a process in the locations and interactions among different sources, both within and outside the cells.

The adaptation mechanisms involve a multistep cellular mechanotransduction process, including: (i) mechanosensing - conversion of mechanical forces into local mechanical signals, such as fluid shear stresses, which initiate a cellular response and (ii) mechanosignaling transduction of an intracellular signaling event occurring in response to a mechanical force and gene expression or protein activation, which ultimately alters cell phenotype and function (Argentati et al., 2019; Ingber, 2018; Mammoto et al., 2013; Sun et al., 2012) (Figure 3 ). Cells probe and respond to the forces of their surroundings through cytoskeletal networks composed of actin filaments (F-actin), myosin motors (Jégou and Romet-Lemonne, 2021; Lenormand et al., 2007; Matthews et al., 2006). F-actin filaments are assembled with the assistance of actin nucleators, such as formins and Arp2/3, which are involved in generating long unbranched and branched actin filaments, respectively (Rotty et al., 2013; Zalevsky et al., 2001). Furthermore, the myosin-driven contraction of the actin cytoskeleton plays a central role in the ability of stress fibers to sense matrix mechanics and generate contractile forces inside cells (Weirich et al., 2021). Both externally-applied and cell-generated mechanical forces are transmitted across the cytoskeleton to the nucleus, where they alter the epigenetic state and expression of genes relating to cellular homeostasis to maintain self-renewal and pluripotency (Li et al., 2020; Vining and Mooney, 2017). Based on these mechanisms, the intracellular processes favoring the interpretation of mechanical cues can be classified here. In this context, the first step is universally recognized as the change in cell behaviors, such as cell-cell interaction, cell-substrate interaction, and cell migration, which serves as the central trigger, converting these input signals into cellular outputs, which in turn manifest as changes to the actin cytoskeleton of the cells. In addition, the actin-myosin cytoskeleton is a major "integrator" and "organizer" of mechanical and biochemical signaling inputs that continuously senses, organizes, and integrates these signals using its control "effector" protein belonging to the Rho family of GTPases (Arnold et al., 2017; Kimura et al., 1996). Specially, the antagonistic activities of RhoA and Rac1 GTPases play a role in the dynamics of myosin-mediated contraction and relaxation during cell migration in several cellular settings (Thanuthanakhun et al., 2021). This confirms that the cells adapt to culture environment through the alteration of the Rho-Rho kinase-phospho-myosin pathway, influencing the epigenetic modifications and the transcription factors that contribute to their inherent states (Thanuthanakhun et al., 2021). Studies using ESCs and iPSC cultures revealed that the time-dependent regulation of two key bivalent epigenetic marks, histone $\mathrm{H} 3$ trimethylation at lysine 4 (H3K4me3) and histone $\mathrm{H} 3$ trimethylation at lysine 27 (H3K27me3), could contribute to the maintenance of the stem cell state and differentiation potential (Harikumar and Meshorer, 2015; Li et al., 2018; Thanuthanakhun et al., 2021). This regulation can take place through epigenetic changes and may be manifested as a "memorizer." Memories acquired during culture are initialized by the disruption of the actin cytoskeleton during enzymatic digestion-based passage culture while maintaining cell homeostasis. 
Thus, by adopting these definitions, we can describe the intracellular processes favoring the interpretation of mechanical cues and concentric groups acting to deliver the signals from cell surface receptors in the plasma membrane to the nucleus via cytoskeleton (Figure 4 ). This chain reaction can eventually lead to epigenetic changes and may be manifested as "memorizer." These multiple correlations can be described using the key factors "trigger," "effector," and "memorizer," and the impact of mechanical force application to the cells may be best described using the terms "sensory," "short-term," and "long-term" memory, implying that any of the various definitions or various components can be used to describe different coordinating systems. The short-term memory induced during growth processes is transient and repeatedly undergoes initialization and re-formation during the passage culture. However, the acquired long-term memory is permanent and is stored or consolidated after passage culture. This simple conceptual framework and the comprehensive classification approach described here provides potential mechanistic insights into the formation and maintenance of epigenetic memory for lasting changes in cell behavior during different growth phases, and indicates that the appropriate seeding density and time required to maintain cellular homeostasis are important from a technological perspective for developing and optimizing bioprocess design and operation.

\section{Mechanobiological Conceptual Framework for Assessment and Prediction of Stem Cell Bio- process: A Case Study}

To understand how cells control and exploit the extent of intrinsic disorder in bioprocesses, we must develop a conceptual framework to define the objective of the assessment. Here, we illustrate a conceptual framework with example case studies and discuss about future outlooks and challenges on the use of data-driven approaches (Figure 5 ). The comparison between intrinsic disorder sources highlights how the consensus on biological indicators of intrinsic disorder captures the main features of intrinsic disorder and correlates well with growth kinetic profiles. Growth kinetics studies are often used to optimize bioprocesses because cells respond to external disturbances by maintaining a homeostatic level of mechanical tension, and these studies lead to the establishment of specific criteria for cell and tissue production (Ehrig et al., 2019; Kato et al., 2018; Nath et al., 2018). In many stem cell studies, it has been reported that the ability to adjust growth kinetics in response to unpredictable environmental fluctuations is associated with the impacts of mechanical force changes (Borys et al., 2021; Kato et al., 2018; Nath et al., 2018). Four basic shapes are hypothesized as plausible to represent the dominant growth curve shape experienced by bioengineers during the culture period. There are four different types of growth: time-dependent, time-delay, tardive, and uncertainty. The identification of phenomena related to the quality of the growth kinetics analysis depends directly on the processing applied to the initial and subsequent data. The use of growth kinetics analysis in the estimation of the impact of study interventions not only improves the statistical quality of the estimates but also enables rational process optimization alongside the dynamic changes of cell requirements throughout the process progression.

For successful development and optimization of stem cell bioprocesses, we must study the fluctuations in current bioprocesses and the variability in stem cells and derived products allowed by regulators and their applications to obtain an understanding of improvement areas (Kim and Kino-oka, 2020a; Kim and Kino-oka, 2020b). The main problem is the lack of biological indicators that use formulas to facilitate their assessment and comparability of bioprocess effectiveness for various external stimuli. Thus, the designed classifier based on mechanical signals will help understand the relationship between externally forced and internally generated variations and identify the time-varying noise generation within and between processes. Based on mechanobiological conceptual frameworks, identifying and quantifying the relationship between inputs, outputs, and changes in bioprocess properties can provide deep insights into bioprocess interactions and would allow the opportunity to balance the costs and benefits of cell production while explicitly acknowledging and internalizing the unintended outcomes of bioprocess strategies. The proposed indicators for assessing a fluctuating interaction network and time-varying stability and consistency are used together with target or threshold values of the metrics employed for determining the degree of stability of a bioprocess responding to externally applied forces. The discussion on which biological indicators are more suitable for describing the cause of intrinsic disorders in stem cell bioprocesses and predicting the impact of mechanical forces in nature and engineered systems is still ongoing. The processes should fit within an overall stem cell-based 
bioprocess, and where current automation is capable of replicating manual processes, if we consider future production systems there must be a move towards machine tools that are more advanced than a human manual process (Ratcliffe et al., 2011). The determination of growth kinetics, thus, paved a way for the optimal design of the operating conditions and process for optimal product formation. Acceptable ranges for a subset of these indicators were established based on a combination of mechanical, biological, and clinical studies and prior knowledge. The determination of the boundary conditions of applied mechanical forces is important to ascertain robust and reproducible cell culture processes that result in the right product quality and suitable product yields. The proposed conceptual framework here will help reduce process variability and increase within- and between-process reproducibility by understanding bioprocess forces and cellular responses of interest and will ensure that the calibrated apparatus performance checks are carried out and operation limits are set adequately.

\section{Concluding Remarks and Future Perspectives}

A mechanobiological conceptual framework with quantification of uncertainties is required for designing and optimizing stem cell bioprocesses to improve process performance, reduce process variability, and ensure consistent product quality. Based on the knowledge of potential sources of noise and variability in a bioprocess, it is possible to systematically categorize the main sources of intrinsic disorder at the bioprocess site and cellular noise and, therefore, act directly to reduce them. Here, we focused on recent advances in the development of stem cell mechanobiology and discussed the necessity for mechanobiological consideration in supporting the development and implementation of successful bioprocessing. We discussed the necessity for and the design principles of a criteria and indicators for assessing the intrinsic disorder in continuous bioprocessing. With the help of the conceptual framework, we determined the effects of the input variability on the performance indicators and compared them between the operations, as well as identified the mode most robust against the input variations. The proposed criteria and indicators based on mechanobiological considerations in this review ensure the coverage of many growth patterns under uncertainty and identify biological indicators important for a wide range of processes that are reflected in bioprocess efficiency. The effectiveness and applicability of this new proposed conceptual framework and methodology are demonstrated through a case study. The conceptual framework-based optimization of bioprocess consists of three main steps: (i) identification of culture operation parameters, (ii) identification of associated risks and requirements for risk mitigation, and (iii) translation of operation parameters into technical requirements (Figure 5 ).The assessment and prediction indicators of bioprocess effectiveness link in vitro attributes, such as combination with the existing indicators for cell productivity and survival during cell-based production process, with the working proposal for an improved assessment of stem cell potency, and thus provide a basis for establishing the cell quality prior to their therapeutic application. This procedure can be repeated iteratively at the design stage of each operating procedure, and the best process control trajectories can be developed by numerical optimization techniques. This allows for getting closer to the optimal bioprocess by solving problems related to intrinsic disorder at the continuous bioprocessing site.

Although many questions regarding quantitative evaluation tools and technologies in multi-stage stem cell bioprocessing remain unanswered, we hope that using such a conceptual framework can make the bioprocess design even more rational and quantitative. Since a conceptual framework can guide research by providing a visual representation of theoretical constructs and variables of interest, this approach can potentially be characterized more effectively and predicted more accurately. Its functionality can be maximized, and engineering specifications imposed by clinical or industrial translation will hopefully present a lower barrier, improving the chances for the translational success of bioprocesses. The technological and computational advances have facilitated the quantification of mRNA and protein level under different simulation environments; however, major challenges remain associated with robust measurement, statistical analysis, and experimental validation. The application of these new mechanical force estimation technologies along with biological indicators provides an opportunity to both learn new strategies for stem cell bioprocessing and educate others on new ways of implementing these technologies. Altogether, proposed conceptual framework will play an increasingly important role in stem cell bioprocesses, which will ultimately lead to the application of models in several fields of the process development chain as well more advanced automation and 
integrated process development in the near future.

\section{ACKNOWLEDGMENTS}

This work was supported by Project Focused on Developing Key Evaluation Technology: Development of Platform Technology for Drug Discovery through Application of Regenerative Medicine from AMED under Grant Number JP19be0604001.

\section{CONFLICT OF INTERESTS}

The authors declare that they have no competing interests.

\section{AUTHORS CONTRIBUTION}

Mee-Hae Kim: original draft preparation, visualization, conceptualization, review and editing; Masahiro Kino-oka: conceptualization, review and editing, funding acquisition

\section{ORCID}

Mee-Hae Kim https://orcid.org/0000-0001-5661-9249

Masahiro Kino-oka https://orcid.org/0000-0002-4912-5811

\section{REFERENCES}

Alisafaei, F., Jokhun, D.S., Shivashankar, G.V., \& Shenoy, V. B. (2019). Regulation of nuclear architecture, mechanics, and nucleocytoplasmic shuttling of epigenetic factors by cell geometric constraints. Proceedings of the National Academy of Sciences, 116 , 13200-13209. https://doi: 10.1073/pnas.1902035116

Ambriz, X., de Lanerolle, P., Ambrosio, J. R. (2018). The mechanobiology of the actin cytoskeleton in stem cells during differentiation and interaction with biomaterials.Stem Cells International, 2018 , 2891957. https://doi: 10.1155/2018/2891957

Argentati, C., Morena, F., Tortorella, I., Bazzucchi, M., Porcellati, S., Emiliani, C., \& Martino, S. (2019). Insight into mechanobiology: How stem cells feel mechanical forces and orchestrate biological functions. International Journal of Molecular Sciences , 20 , 5337. https://doi: 10.3390/ijms20215337

Arnold, T.R., Stephenson, R.E., \& Miller, A.L. (2017). Rho GTPases and actomyosin: Partners in regulating epithelial cell-cell junction structure and function. Experimental Cell Research, 358 , 20-30. https://doi: 10.1016/j.yexcr.2017.03.053

Borys, B.S., Dang, T., So, T., Rohani, L., Revay, T., Walsh, T., Thompson, M., Argiropoulos, B., Rancourt, D.E., Jung, S., Hashimura, Y., Lee, B., \& Kallos, M.S. (2021). Overcoming bioprocess bottlenecks in the large-scale expansion of high-quality hiPSC aggregates in vertical-wheel stirred suspension bioreactors. Stem Cell Research $\mathscr{E}$ Therapy, 12, 55. https://doi: 10.1186/s13287-020-02109-4.

Charras, G., \& Yap, A.S. (2018). Tensile Forces and Mechanotransduction at cell-cell junctions. Current Biology, 28 , R445-R457. https://doi: 10.1016/j.cub.2018.02.003

Chen, L., Huang, T., Qiao, Y., Jiang, F., Lan, J., Zhou, Y., Yang, C., Yan, S., Luo, K., Su, L., \& Li, J. (2019). Perspective into the regulation of cell-generated forces toward stem cell migration and differentiation. Journal of Cellular Biochemistry, 120 , 8884-8890. https://doi: 10.1002/jcb.28251

Discher, D., Dong, C., Fredberg, J.J., Guilak, F., Ingber, D., Janmey, P., Kamm, R.D., Schmid-Schönbein, G.W., \& Weinbaum, S. (2009). Biomechanics: cell research and applications for the next decade. Annals of Biomedical Engineering, 37 , 847-859. https://doi: 10.1007/s10439-009-9661-x

Dupont, S., Morsut, L., Aragona, M., Enzo, E., Giulitti, S., Cordenonsi, M., Zanconato, F., Le Digabel, J., Forcato, M., Bicciato, S., Elvassore, N., \& Piccolo, S. (2011). Role of YAP/TAZ in mechanotransduction. Nature, 474 , 179-183. https://doi: 10.1038/nature10137 
Ehrig, S., Schamberger, B., Bidan, C.M., West, A., Jacobi, C., Lam, K., Kollmannsberger, P., Petersen, A., Tomancak, P., Kommareddy, K., Fischer, F.D., Fratzl, P., \& Dunlop, J.W.C. (2019). Surface tension determines tissue shape and growth kinetics. Science Advances, 5,eaav9394. https://doi: 10.1126/sciadv.aav9394

Freund, J. B., Goetz, J. G., Hill, K. L., \& Vermot, J. (2012). Fluid flows and forces in development: functions, features and biophysical principles. Development, 139 , 1229-1245. https://doi: 10.1242/dev.073593

Goodwin, K., \& Nelson, C.M. (2021). Mechanics of development. Developmental Cell, 56, 240-250. https://doi: 10.1016/j.devcel.2020.11.025

Harikumar, A., \& Meshorer, E. (2015). Chromatin remodeling and bivalent histone modifications in embryonic stem cells. EMBO reports, 16 , 1609-1619. https://doi: 10.15252/embr.201541011.

Harrison, R.P., Medcalf, N., \& Rafiq, Q.A. (2018). Cell therapy-processing economics: small-scale microfactories as a stepping stone toward large-scale macrofactories.Regenerative Medicine, 13 , 159-173. https://doi: 10.2217/rme-2017-0103

Hilfinger, A. \& Paulsson, J. (2011). Separating intrinsic from extrinsic fluctuations in dynamic biological systems. Proceedings of the National Academy of Sciences, 108, 12167-12172, https://doi.org/10.1073/pnas.1018832108

Ingber, D.E. (1997). Tensegrity: the architectural basis of cellular mechanotransduction. Annual Review of Physiology, 59 , 575- 599. https://doi: 10.1146/annurev.physiol.59.1.575

Ingber, D.E. (2018). From mechanobiology to developmentally inspired engineering. Philosophical Transactions of The Royal Society B Biological Sciences, 373 , 20170323. https://doi: 10.1098/rstb.2017.0323

Ivanovska, I.L., Shin, J.W., Swift, J., \& Discher, D. (2015). Stem cell mechanobiology: diverse lessons from bone marrow. Trends in Cell Biology, 25, 523-532. https://doi: 10.1016/j.tcb.2015.04.003

Iskratsch, T., Wolfenson, H., \& Sheetz, M. P. (2014). Appreciating force and shape - the rise of mechanotransduction in cell biology. Nature Reviews Molecular Cell Biology, 15 , 825-833. https://doi: 10.1038/nrm3903

Jégou, A. \& Romet-Lemonne, G. (2021). Mechanically tuning actin filaments to modulate the action of actin-binding proteins. Current Opinion in Cell Biology, 68 , 72-80. https://doi: 10.1016/j.ceb.2020.09.002

Jo, J., Abdi Nansa, S., \& Kim, D.H. (2020). Molecular regulators of cellular mechanoadaptation at cell-material interfaces. Frontiers in Bioengineering and Biotechnology, 8 , 608569. https://doi: 10.3389/fbioe.2020.608569

Kato, R., Kim M.H., \& Kino-oka, M. (2018). Comparison of growth kinetics between static and dynamic cultures of human induced pluripotent stem cells. Journal of Bioscience and Bioengineering, 125, 736-740. https://doi: 10.1016/j.jbiosc.2018.01.002

Kinney, M.A., Vo, L.T., Frame, J.M., Barragan, J., Conway, A.J., Li, S., Wong, K.K., Collins, J.J., Cahan, P., North, T.E., Lauffenburger, D.A., \& Daley, G.Q. (2019). A systems biology pipeline identifies regulatory networks for stem cell engineering. Nature Biotechnology, 37 , 810-818. https://doi: 10.1038/s41587-019$0159-2$

Kami, D., Watakabe, K., Yamazaki-Inoue, M., Minami, K., Kitani, T., Itakura, Y., Toyoda, M., Sakurai, T., Umezawa, A., \& Gojo, S. (2013). Large-scale cell production of stem cells for clinical application using the automated cell processing machine. BMC Biotechnology, 13 , 102. https://doi: 10.1186/1472-6750-13-102

Kim, M.H., \& Kino-oka, M. (2018). Bioprocessing strategies for pluripotent stem cells based on Waddington's epigenetic landscape. Trends in Biotechnology, 36 , 89-104. https://doi: 10.1016/j.tibtech.2017.10.006

Kim, M.H. \& Kino-oka, M. (2020a). Bioengineering considerations for a nurturing way to enhance scalable expansion of human pluripotent stem cells. Biotechnology Journal, 15, e1900314. https://doi: 10.1002/biot.201900314 
Kim, M.H. \& Kino-oka, M. (2020b). Designing a blueprint for next-generation stem cell bioprocessing development. Biotechnology and Bioengineering, 117 , 832-843. https://doi: 10.1002/bit.27228

Kimura, K., Ito, M., Amano, M., Chihara, K., Fukata, Y., Nakafuku, M., Yamamori, B., Feng, J., Nakano, T., Okawa, K., Iwamatsu, A., \& Kaibuchi, K. (1996). Regulation of myosin phosphatase by Rho and Rhoassociated kinase (Rho-kinase). Science 273, 245-248. https://doi: 10.1126/science.273.5272.245

Lei J. (2009). Stochasticity in single gene expression with both intrinsic noise and fluctuation in kinetic parameters. Journal of Theoretical Biology, 256, 485-492. https://doi: 10.1016/j.jtbi.2008.10.028.

Lenormand, G., Bursac, P., Butler, J.P., \& Fredberg, J.J. (2007). Out-of-equilibrium dynamics in the cytoskeleton of the living cell.Physical Review E, 76 , 41901. https://doi: 10.1103/PhysRevE.76.041901

Li, F., Wan, M., Zhang, B., Peng, Y., Zhou, Y., Pi, C., Xu, X., Ye, L., Zhou, X., \& Zheng, L. (2018). Bivalent histone modifications and development. Current Stem Cell Research ES Therapy, 13, 83-90. https://doi: 10.2174/1574888X12666170123144743

Li, J., Liu, Y., Zhang, Y., Yao, B., Enhejirigala, Li, Z., Song, W., Wang, Y., Duan, X., Yuan, X., Fu, X., \& Huang, S. (2021). Biophysical and biochemical cues of biomaterials guide mesenchymal stem cell behaviors. Frontiers in Cell and Developmental Biology, 9 , 640388. https://doi: 10.3389/fcell.2021.640388

Li, S., Yang, D., Gao, L., Wang, Y., \& Peng, Q. (2020). Epigenetic regulation and mechanobiology. Biophysics Reports, 6 , 33-48. https://doi.org/10.1007/s41048-020-00106-x

Liu, L., Yuan, W., \& Wang, J. (2010). Mechanisms for osteogenic differentiation of human mesenchymal stem cells induced by fluid shear stress. Biomechanics and Modeling in Mechanobiology, 9 , 659-670. doi: 10.1007/s10237-010-0206-x

Mack, P. J., Kaazempur-Mofrad, M. R., Karcher, H., Lee, R. T., \& Kamm, R. D. (2004). Force-induced focal adhesion translocation: effects of force amplitude and frequency. American Journal of Physiology, 287 , C954-C962. https://doi: 10.1152/ajpcell.00567.2003

Mizuno, D., Tardin, C., Schmidt, C. F., \& Mackintosh, F. C. (2007). Nonequilibrium mechanics of active cytoskeletal networks. Science, 315(5810):370-373. https://doi: 10.1126/science.1134404

Mount, N.M., Ward, S.J., Kefalas, P., \& Hyllner, J. (2015). Cell-based therapy technology classifications and translational challenges. Philosophical Transactions of the Royal Society, 370, 20150017. https://doi: 10.1098/rstb.2015.0017

Matthews, B. D., Overby, D. R., Mannix, R. \& Ingber, D. E. (2006). Cellular adaptation to mechanical stress: role of integrins, Rho, cytoskeletal tension and mechanosensitive ion channels. Journal of Cell Science, 119 , 508-518. https://doi: $10.1242 /$ jcs. 02760

Maul, T.M., Chew, D.W., Nieponice, A., \& Vorp, D.A. (2011). Mechanical stimuli differentially control stem cell behavior: morphology, proliferation, and differentiation. Biomechanics and Modeling in Mechanobiology, 10 , 939-953. https://doi: 10.1007/s10237-010-0285-8

Mammoto, T., Mammoto, A., \& Ingber, D.E. (2013). Mechanobiology and developmental control. Annual Review of Cell and Developmental Biology, 29 , 27-61. https://doi: 10.1146/annurev-cellbio-101512-122340

Mao, Y. \& Baum, B. (2015). Tug of war-the influence of opposing physical forces on epithelial cell morphology. Developmental Biology, 401 , 92-102. https://doi: 10.1016/j.ydbio.2014.12.030

Madl, C.M., Heilshorn, S.C., Blau, H.M. (2018). Bioengineering strategies to accelerate stem cell therapeutics. Nature, 557 , 335-342. https://doi: 10.1038/s41586-018-0089-z

Mack, P. J., M. R. Kaazempur-Mofrad, H. Karcher, R. T. Lee,. \& R. D. Kamm. (2004). Force-induced focal adhesion translocation: effects of force amplitude and frequency. American Journal of Physiology, 287 , C954-C962. https://doi: 10.1152/ajpcell.00567.2003 
McKee, C. \& Chaudhry, G.R. (2017). Advances and challenges in stem cell culture. Colloids and Surfaces B: Biointerfaces, 159 , 62-77. https://doi: 10.1016/j.colsurfb.2017.07.051

Mendez, M.G., \& Janmey, P.A. (2012). Transcription factor regulation by mechanical stress. International Journal of Biochemistry and Cell Biology, 44, 728-32. https://doi: 10.1016/j.biocel.2012.02.003

Mendelson, A. \& Frenette, P.S. (2014). Hematopoietic stem cell niche maintenance during homeostasis and regeneration. Nature Medicine, 20, 833-846. https://doi: 10.1038/nm.3647

Miroshnikova, Y. A., Nava, M. M., \& Wickstrom, S. A. (2017). Emerging roles of mechanical forces in chromatin regulation. Journal of Cell Science, 130 , 2243-2250. https://doi: 10.1242/jcs.202192

Misener, R., Allenby, M.C., Fuentes-Gari, M., Gupta, K., Wiggins, T., Panoskaltsis, N., Pistikopoulos, E.N., \& Mantalaris, A. (2018). Stem cell biomanufacturing under uncertainty: A case study in optimizing red blood cell production. AIChE Journal, 64 , 3011-3022. https://doi: 10.1002/aic.16042

Kaazempur Mofrad, M.R., Abdul-Rahim, N.A., Karcher, H., Mack, P.J., Yap, B., \& Kamm, R.D. (2005). Exploring the molecular basis for mechanosensation, signal transduction, and cytoskeletal remodeling. Acta Biomaterialia, 1 , 281-293. https://doi: 10.1016/j.actbio.2005.02.008.

Naqvi, S.M. \& McNamara, L.M. (2020). Stem cell mechanobiology and the role of biomaterials in governing mechanotransduction and matrix production for tissue regeneration. Frontiers in Bioengineering and Biotechnology, 8 , 597661. https://doi: 10.3389/fbioe.2020.597661

Nath, S.C., Tokura, T., Kim M.H., \& Kino-oka M. (2018). Botulinum hemagglutinin-mediated in situ breakup of human induced pluripotent stem cell aggregates for high-density suspension culture.Biotechnology and Bioengineering, 115, 910-920. https://doi: 10.1002/bit.26526

Panchalingam, K.M., Jung, S., Rosenberg, L., \& Behie, L.A. (2015). Bioprocessing strategies for the largescale production of human mesenchymal stem cells: a review. Stem Cell Research \&5 Therapy, 6 , 225. https://doi: 10.1186/s13287-015-0228-5

Pittenger, M.F., Mackay, A.M., Beck, S.C., Jaiswal, R.K., Douglas, R., Mosca, J.D., Moorman, M.A., Simonetti, D.W., Craig, S., \& Marshak, D.R. (1999a). Multilineage potential of adult human mesenchymal stem cells. Science, 284 , 143-147. https://doi: 10.1126/science.284.5411.143

Pittenger, M.F., Discher, D.E., Péault, B.M., Phinney, D.G., Hare, J.M., \& Caplan, A.I. (2019) Mesenchymal stem cell perspective: cell biology to clinical progress. NPJ Regenerative Medicine, 4, 22. https://doi: $10.1038 / \mathrm{s} 41536-019-0083-6$

Ratcliffe, E., Thomas, R.J., \& Williams, D.J. (2011). Current understanding and challenges in bioprocessing of stem cell-based therapies for regenerative medicine. British Medical Bulletin, 100, 137-155. https://doi: $10.1093 / \mathrm{bmb} / \mathrm{ldr} 03$

Raser, J. M., \& O'shea, E. K. (2005). Noise in gene expression: origins, consequences, and control. Science, 309 , 2010-2013, https://doi.org/10.1126/science.1105891

Roca-Cusachs, P., Conte, V., \& Trepat, X. (2017). Quantifying forces in cell biology. Nature Cell Biology, 19, 742-751. https://doi: 10.1038/ncb3564

Rosowski, K.A., Schreiner, S.M., Horsley, V., \& King, M.C. (2015). Nuclear-cytoskeletal linkages facilitate cross talk between the nucleus and intercellular adhesions. Journal of Cell Biology, 209 , 403-418. https://doi: $10.1083 /$ jcb.201502024

Rotty, J.D., Wu, C., \& Bear, J.E. (2013). New insights into the regulation and cellular functions of the ARP2/3 complex. Nature Reviews Molecular Cell Biology, 14, 7-12. https://doi: 10.1038/nrm3492

Shuzui, E., Kim, M.H., \& Kino-oka, M. (2019). Anomalous cell migration triggers a switch to deviation from the undifferentiated state in colonies of human induced pluripotent stems on feeder layers. Journal of 
Bioscience and Bioengineering, 127, 246-255. https://doi: 10.1016/j.jbiosc.2018.07.020

Soltani, M., Vargas-Garcia, C.A., Antunes, D., \& Singh, A. (2016). Intercellular variability in protein levels from stochastic expression and noisy cell cycle processes. PLOS Computational Biology, 12, e1004972. https://doi: 10.1371/journal.pcbi.1004972

Stricker, J., Falzone, T., \& Gardel, M.L. (2010). Mechanics of the F-actin cytoskeleton. Journal of Biomechanics, 43 , 9-14. https://doi: 10.1016/j.jbiomech.2009.09.003

Sun, Y., Chen, C.S., \& Fu, J. (2012). Forcing stem cells to behave: a biophysical perspective of the cellular microenvironment. Annual Review of Biophysics, 41 , 519-542. https://doi: 10.1146/annurev-biophys042910-155306

Swain, P.S., Elowitz, M.B., \& Siggia, E.D. (2002). Intrinsic and extrinsic contributions to stochasticity in gene expression.Proceedings of the National Academy of Sciences, 99,12795-12800. https://doi: 10.1073/pnas.162041399

Tajik, A., Zhang, Y., Wei, F., Sun, J., Jia, Q., Zhou, W., Singh, R., Khanna, N., Belmont, A.S., \& Wang, N. (2016). Transcription upregulation via force-induced direct stretching of chromatin. Nature Materials, 15 , 1287-1296. https://doi: 10.1038/nmat4729

Tewary, M., Shakiba, N., \& Zandstra, P.W. (2018). Stem cell bioengineering: building from stem cell biology. Nature Reviews Genetics, 19 , 595-614. https://doi: 10.1038/s41576-018-0040-z

Thanuthanakhun, N., Kino-oka, M., Borwornpinyo, S., Ito, Y., \& Kim, M.H. (2021). The impact of culture dimensionality on behavioral epigenetic memory contributing to pluripotent state of iPS cells. Journal of Cellular Physiology, 236 , 4985-4996. https://doi: 10.1002/jcp.30211

Thomas, P. (2019). Intrinsic and extrinsic noise of gene expression in lineage trees. Scientific Reports, 9, 474. https://doi: 10.1038/s41598-018-35927-x.

Thomson, J.A., Itskovitz-Eldor, J., Shapiro, S.S., Waknitz, M.A., Swiergiel, J.J., Marshall, V.S., \& Jones, J.M. (1998). Embryonic stem cell lines derived from human blastocysts. Science, 282 , 1145-1147. https://doi: 10.1126/science.282.5391.1145

Thomas, P., Terradot, G., Danos, V. \& Weiße, A. Y. (2018). Sources, propagation and consequences of stochasticity in cellular growth.Nature Communications, 9 , 4528, https://doi.org/10.1038/s41467-018-06912-9

Thomas, R.J., Hourd, P., \& Williams, D.J. (2008). Application of process quality engineering techniques to improve the understanding of the in vitro processing of stem cells for therapeutic use. Journal of Biotechnology, 136 , 148-155. https://doi: 10.1016/j.jbiotec.2008.06.009

Tottori, T., Fuji, M., \& Kuroda, S. (2019). Robustness against additional noise in cellular information transmission. Physical Review E, 100 , 042403. https://doi: 10.1103/PhysRevE.100.042403

Vining, K.H., \& Mooney, D.J. (2017). Mechanical forces direct stem cell behaviour in development and regeneration. Nature Reviews Molecular Cell Biology , 18, 728-742. https://doi: 10.1038/nrm.2017.108

Walker, M., Godin, M., Harden, J.L., \& Pelling, A.E. (2020a). Time dependent stress relaxation and recovery in mechanically strained 3D microtissues. APL Bioengineering, 4 , 036107. https://doi: 10.1063/5.0002898

Walker, M., Rizzuto, P., Godin, M., \& Pelling, A.E. (2020b). Structural and mechanical remodeling of the cytoskeleton maintains tensional homeostasis in 3D microtissues under acute dynamic stretch. Scientific Reports, 10 , 7696. https://doi: 10.1038/s41598-020-64725-7

Wall, M., Butler, D., El Haj, A., Bodle, J.C., Loboa, E.G., \& Banes, A.J. (2018). Key developments that impacted the field of mechanobiology and mechanotransduction. Journal of Orthopaedic Research, 36 , 605619. https://doi: 10.1002/jor.23707 
Webster, K.D., Ng, W.P., \& Fletcher, D.A. (2014). Tensional homeostasis in single fibroblasts. Biophysical Journal, 107, 146-155. https:// doi: 10.1016/j.bpj.2014.04.051

Weirich, K.L., Stam, S., Munro, E., \& Gardel, M.L. (2021). Actin bundle architecture and mechanics regulate myosin II force generation.Biophysical Journal, 120 , 1957-1970. https://doi: 10.1016/j.bpj.2021.03.026

Yamanaka S. (2020). Pluripotent stem cell-based cell therapy-promise and challenges. Cell Stem Cell, 27 , 523-531. https://doi: 10.1016/j.stem.2020.09.014

Zalevsky, J., Lempert, L., Kranitz, H., \& Mullins, R.D. (2001). Different WASP family proteins stimulate different Arp2/3 complex-dependent actin-nucleating activities. Current Biology, 11, 1903-1913. https://doi: 10.1016/s0960-9822(01)00603-0

Zhu, Y., Ge, J., Huang, C., Liu, H., \& Jiang, H. (2021). Application of mesenchymal stem cell therapy for aging frailty: from mechanisms to therapeutics. Theranostics, 11 , 5675-5685. https://doi: 10.7150/thno. 46436

\section{Figure Legends}

Fig. 1. Molecular mechanisms of mechanotransduction in stem cells. Mechanotransduction describes the ability of cells to sense mechanical forces and translate them into biochemical reactions for signal transduction and biological responses. When external forces, such as fluid shear stress, are applied to cells, these forces are transferred from the ECM to the cell, propagating from the outside into the cell. Integrins are a family of transmembrane receptors connecting the cytoskeleton to the ECM. They are also known to initiate cytosolic signaling upon binding to extracellular ligands (outside-in signaling) as well have their binding affinity regulated intracellularly (inside-out signaling). Several mechanosensor proteins, including mechanosensing ion channels, cell-substrate and cell-cell junctional complexes, and cytoskeleton-associated complexes, have been identified in stem cells. Arrows and bars represent the net result of a signaling pathway. Abbreviations used: ECM, extracellular matrix; RhoA, Rho kinase; ROCK, Rho-associated protein kinase; LINC, linker of nucleoskeleton and cytoskeleton.

Fig. 2. Understanding the internal and external variability-generated intrinsic disorder in stem cell bioprocess based on mechanobiological considerations. The cell responses to internal and external forces are represented by the time-related phenomena in biological processes; time-dependency, time-delay, tardive, and uncertainty. These may lead to cellular bioprocess variability, the so-called intrinsic disorder that affects bioprocess efficiency.

Fig. 3. A simplified view of mechanosensing and mechanotransduction signaling pathways for regulating stem cell homeostasis. Cellular behavior is affected by mechanical forces through the process of mechanotransduction, in which mechanical stimuli from their extracellular environment or cell-generated forces are converted into a set of biochemical reactions and a cellular response. We categorized the "intrinsic cellular processes" as "trigger," "effector," and "memorizer" key factors involved in mechanotransduction. The actin-myosin cytoskeleton is defined as the "integrator" and "organizer" of mechanical and biochemical signaling inputs in these processes. Arrows and bars represent the net result of a signaling pathway. Arrows and bars represent the net result of a signaling pathway. Abbreviations used: RhoA, Rho kinase; MLC-P, phosphorylated myosin II regulatory light chain; MLC-P, myosin light chain phosphatase; MLCK, myosin light chain kinase.

Fig. 4. Development of criteria and indicators applicable to the study of stem cell bioprocess effectiveness based on mechanobiological mechanisms. We propose new indicators to assess the cell status associated with cell behavior-driven epigenetic memory formation in human induced pluripotent stem cell (hiPSC) culture, as a case study. The acquired memories are transformed from a short-term memory into a long-term memory in transition between exponential and long-term stationary phases. The relationship between the indicators in the same category was used to determine the cell state and differentiation potential. The combination and integration of qualitative and quantitative methods can be used to enhance stem cell bioprocess optimization without compromising the product quality. 
Fig. 5. Potential strategies and practical considerations to stem cell bioprocess optimization with the conceptual framework based on mechanobiological mechanisms. We propose a new conceptual framework that systematically links three dimensions: inputs, outputs, and the associated system level outcomes of bioprocess effectiveness. Based on the dynamic relationship between the key factors (trigger, effector, and memorizer) affecting the formation of epigenetic memory (sensory, short-term, and long-term), the determination of the boundary conditions of applied mechanical forces is important to enhance cell quality and process robustness. The application of the conceptual frameworks can apply an important approach that facilitate movement to understand, predict, and control the intrinsic disorder in stem cell bioprocessing. Finally, this allows that the output of the design process, including a quality control plan, a bioprocessing plane, and a strategy for facilities, validation, and other requirements.

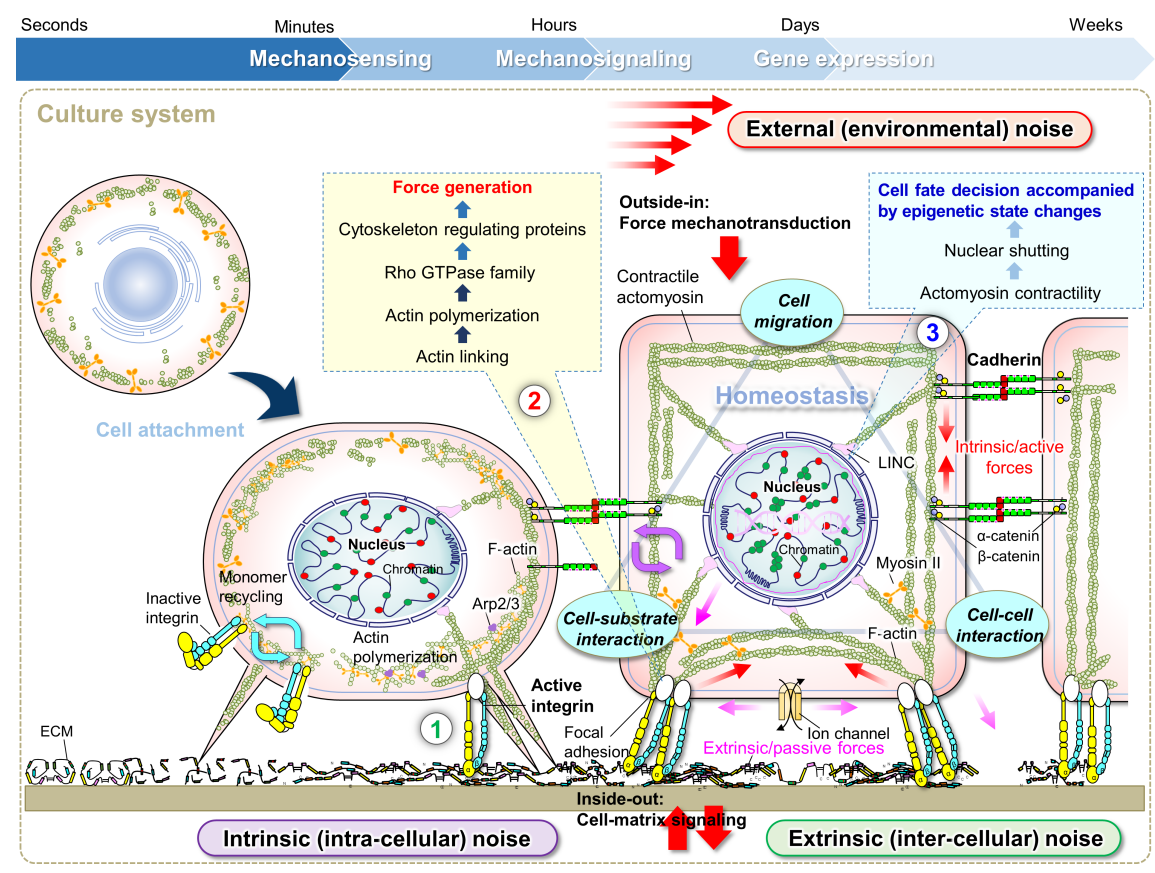



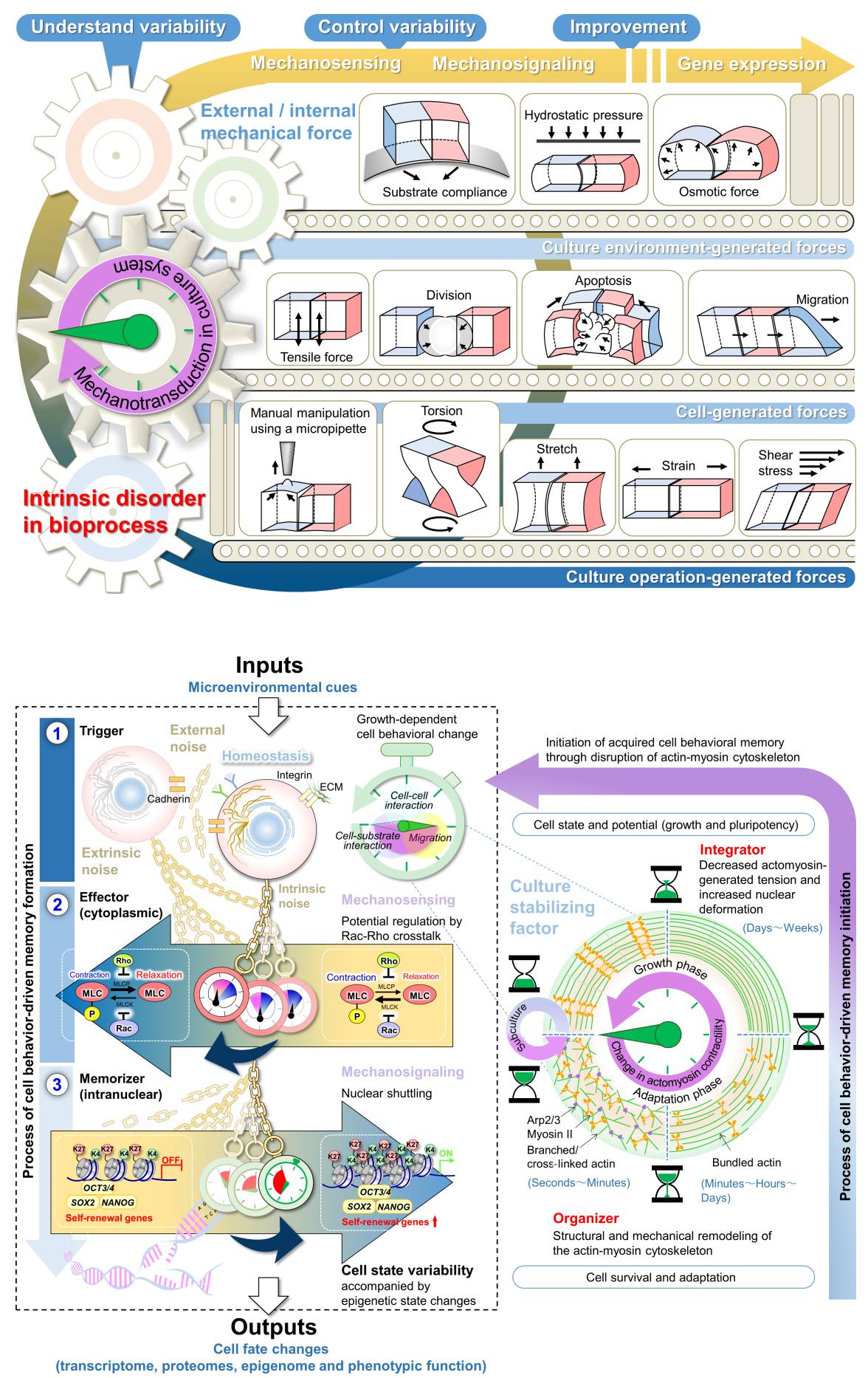

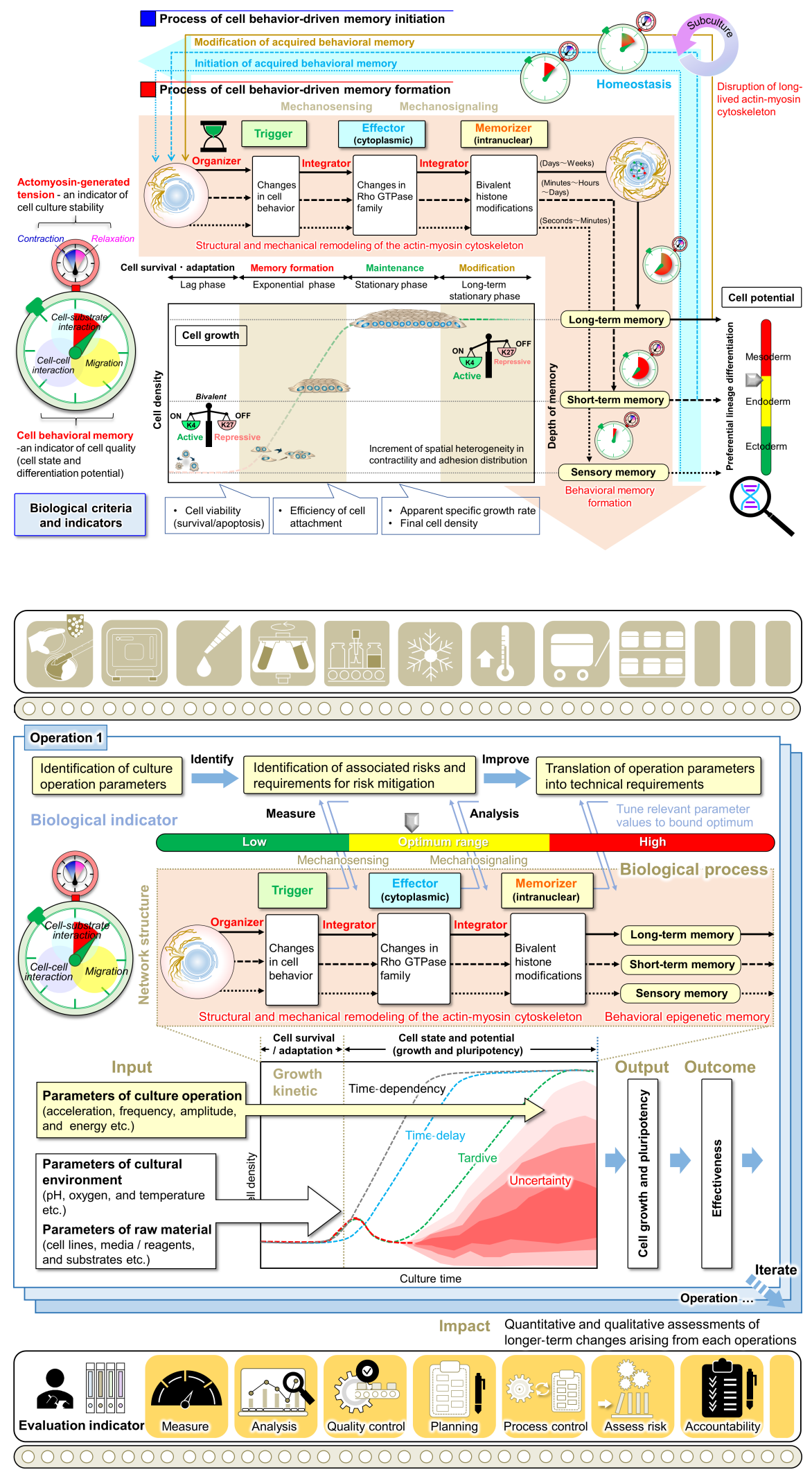